\title{
Washington photometry of candidate star clusters in the Small Magellanic Cloud
}

\author{
Andrés E. Piatti ${ }^{1 \star}$ and Eduardo Bica ${ }^{2}$ \\ ${ }^{1}$ Instituto de Astronomía y Física del Espacio, CC 67, Suc. 28, 1428 Ciudad de Buenos Aires, Argentina \\ ${ }^{2}$ Departamento de Astronomía, Universidade Federal do Rio Grande do Sul, CP 15051, Porto Alegre, 91500-970, Brazil
}

Accepted 2012 July 10. Received 2012 July 10; in original form 2012 May 22

\begin{abstract}
We present for the first time Washington $C T_{1}$ photometry for 11 unstudied or poorly studied candidate star clusters. The selected objects are of small angular size, contain a handful of stars, and are projected towards the innermost regions of the Small Magellanic Cloud (SMC). The respective colour-magnitude diagrams (CMDs) were cleaned of the unavoidable star field contamination by taking advantage of a procedure which makes use of variable size CMD cells. This method has shown to be able to eliminate stochastic effects in the cluster CMDs caused by the presence of isolated bright stars, as well as to make a finer cleaning in the most populous CMD regions. Our results suggest that nearly one-third of the studied candidate star clusters would appear to be genuine physical systems. In this sense, the ages previously derived for some of them mostly reflect those of the composite stellar populations of the SMC field. Finally, we used the spatial distribution in the SMC of possible non-clusters to statistically decontaminate that of the SMC cluster system. We found that there is no clear difference between expected and observed cluster spatial distributions, although it would become significant at a $2 \sigma$ level between $a \approx 0.3$ and 1.2 (the semi-major axis of an ellipse parallel to the SMC bar and with $b / a=1 / 2$ ), if the asterisms were increased up to 20 per cent.
\end{abstract}

Key words: techniques: photometric - galaxies: individual: SMC - galaxies: star clusters: general.

\section{INTRODUCTION}

The different catalogues of Small Magellanic Cloud (SMC) star clusters have been compiled on the basis of star counts, either by visually inspecting photographic plates (see e.g. Bruck 1975; Hodge 1986; Bica \& Schmitt 1995) or by automatic algorithmic searches (e.g. Pietrzynski et al. 1999). As far as we are aware of, the most recent catalogue that puts all the previous ones together is that of Bica et al. (2008, hereafter B08). Although it is expected that most of the catalogued objects are indeed genuine physical systems, it was beyond the scope of Bica et al. (2008) to verify the physical nature of such faint objects. The task of cleaning cluster catalogues from nonphysical systems or asterisms is far from being an exciting job. Most of the astronomers desire to deal with prominent clusters. For this reason, studies concluding about the asterism or overdensity nature of faint objects in the Clouds are rare or absent. However, those works would be very important and are also required if any statistical analysis about the cluster formation and disruption rates, the cluster spatial, age and metallicity distributions, etc., were attempted.
As it is commonly accepted, an apparent concentration of stars in the sky does not necessarily lead to the conclusion that such concentration constitutes a physical system. The presence of such star concentrations implies that we are dealing with a physical cluster in the case of typical globular clusters or very populous open clusters. For most of the apparent star concentrations in the sky, however, it may be necessary to have supplementary information available about proper motions, radial velocities, spectral types and photometry to confirm their physical reality. The photometric data are often the only information at our disposal from which the existence of a star cluster may be inferred. Even though photometric data are indeed valuable, the steps to conclude on the physical nature of a star aggregate from its colour-magnitude diagram (CMD) might not be a straightforward task. This usually happens when dealing with small objects or sparse clusters projected or immersed in crowded star fields. An example of such situations is those clusters located in the inner regions of the SMC. In such cases, simple circular CMD extraction around the cluster centre could lead to a wrong conclusion, since the CMDs are obviously composed of stars of different stellar populations (Piatti 2012). Consequently, it is hardly possible to assess whether the bright and young main sequence (MS) or the subgiant and red giant branches trace the fiducial cluster features. Glatt, Grebel \& Koch (2010, hereafter 
Table 1. Candidate star clusters in the SMC.

\begin{tabular}{|c|c|c|c|c|c|c|c|c|c|c|c|c|}
\hline \multirow[t]{2}{*}{ Name } & \multirow{2}{*}{$\begin{array}{l}\alpha_{2000} \\
(\mathrm{~h} \mathrm{~m} \mathrm{~s})\end{array}$} & \multirow{2}{*}{$\begin{array}{l}\delta_{2000} \\
\left(0^{\prime \prime \prime}\right)\end{array}$} & \multirow{2}{*}{$\begin{array}{c}l \\
\left({ }^{\circ}\right)\end{array}$} & \multirow{2}{*}{$\begin{array}{c}b \\
\left({ }^{\circ}\right)\end{array}$} & \multirow[t]{2}{*}{ Date } & \multirow{2}{*}{$\begin{array}{l}\text { Exposure } \\
C(\mathrm{~s}) R(\mathrm{~s})\end{array}$} & \multirow{2}{*}{$\begin{array}{l}\text { Airmass } \\
\quad C R\end{array}$} & \multirow{2}{*}{$\begin{array}{c}\text { Seeing } \\
C(\operatorname{arcsec}) R(\operatorname{arcsec})\end{array}$} & \multicolumn{4}{|c|}{$\log ($ age $)$} \\
\hline & & & & & & & & & $\mathrm{Cl}$ & & G1 & \\
\hline B119 & 010419 & -730954 & 301.63 & -43.93 & 2008 December 19 & 1500300 & 1.4161 .403 & 1.20 .9 & - & - & - & - \\
\hline BS25 & 004446 & -725353 & 303.61 & -44.22 & 2008 December 20 & 1500300 & 1.5331 .470 & 1.21 .1 & - & - & - & - \\
\hline BS35 & 004750 & -732842 & 303.28 & -43.64 & 2008 December 20 & 1500300 & 1.5331 .470 & 1.21 .1 & 8.5 & 1 & 8.55 & 1 \\
\hline BS251 & 005126 & -731700 & 302.93 & -43.84 & 2008 December 20 & 1500300 & 1.5331 .470 & 1.21 .1 & 8.4 & 2 & - & - \\
\hline H86-78s & 004612 & -732339 & 303.44 & -43.72 & 2008 December 20 & 1500300 & 1.5331 .470 & 1.21 .1 & - & - & 8.0 & 1 \\
\hline H86-86 & 004701 & -732335 & 303.36 & -43.73 & 2008 December 20 & 1500300 & 1.5331 .470 & 1.21 .1 & - & - & 8.1 & 1 \\
\hline H86-88 & 004658 & -732009 & 303.37 & -43.78 & 2008 December 20 & 1500300 & 1.5331 .470 & 1.21 .1 & 8.8 & 3 & 8.5 & 1 \\
\hline H86-197 & 011530 & -711044 & 300.15 & -45.81 & 2008 December 20 & 1500300 & 1.5331 .470 & 1.21 .1 & - & - & - & - \\
\hline
\end{tabular}

G10) have studied 324 SMC clusters using data from the Magellanic Clouds Photometric Survey (Zaritsky et al. 2002). They show from isochrone fittings on to the CMDs that the studied objects are clusters younger than $1 \mathrm{Gyr}$, mostly distributed in the main body of the galaxy, which is highly crowded. Although they mention that field contamination is a severe effect in the extracted cluster CMDs and therefore influences the age estimates, no decontamination from field CMDs was carried out. It would not be unexpected that some of the studied objects are not real star clusters, particularly those with very uncertain age estimates $[\sigma($ age $) \geq 0.5$ for $\log ($ age $) \lesssim 9.0]$. This possibility alerts us to the fact that solely the circular extraction of the observed CMDs of clusters located in highly populated star fields is not enough neither for an accurate isochrone fitting to the cluster MSs nor for confirming their physical nature.

Different statistical procedures have been proposed with an acceptable success, in order to avoid as much as possible the field contamination in the cluster CMDs. Chiosi et al. (2006, hereafter C06) studied 311 clusters in the central part of the SMC from OGLE data (Udalski et al. 1998) and their own data. They used equivalent cluster areas of fields close to the clusters, but outside the cluster radii, to build field CMDs. Subsequently, they divided the CMDs of both clusters and fields into boxes of size $\Delta(V)=0.5 \mathrm{mag}$ and $\Delta(V-I)=0.2 \mathrm{mag}$, and subtracted for every field star in a box the closest cluster star in the respective box. The cluster ages derived from isochrone fittings on to the cleaned CMDs for 136 clusters, also included in the study of G10, were found to be $\sim 0.2-0.3$ in $\log ($ age $)$ younger than those derived by G10 $[\sigma(\Delta \log ($ age $))=0.13$, age $\lesssim 9.0]$. The main reason for this systematic shift is probably the different metallicities of the isochrones involved. While C06 used the isochrone set of Girardi et al. (2002, $Z=0.008), \mathrm{G} 10$ fitted the cluster CMDs with isochrones computed by Girardi et al. (1995, $Z=0.004)$. Note that for those clusters with the most significant age deviation large age uncertainties are obtained in both C06 and G10. Furthermore, bearing in mind the large age uncertainties quoted by C06 and G10 for some clusters, and that nobody has confirmed that they are genuine physical systems, the doubt about their cluster reality might arise unavoidably.

In this paper, we present an analysis of 11 candidate star clusters from new CCD Washington $C T_{1}$ photometry, in combination with a computational tool for cleaning the star field signature in the cluster CMDs. Our main aim is to be able to confirm the physical reality of the studied objects, once their photometric data have been properly cleaned of field contamination. Indeed, the proposed computational tool for estimating the probability of a star of being an intrinsic feature of the cluster field shows to be able to produce reliable
CMDs revealing the genuine nature of the considered object. Note that the studied objects were catalogued as clusters on the basis of star counts on less deep images than those used in this study. We present the data set in Section 2, while we describe the data handling in Section 3. Section 4 deals, on the one hand, with available star field decontamination procedures and, on the other hand, with the presently used method. In Section 5, our analysis shows that most of these stellar groups are likely genuine star clusters. Finally, we summarize the main results in Section 6.

\section{THE DATA}

Piatti (2012) performed reduction, stellar photometry and photometric standardization of Washington $C T_{1}$ images of $11 \mathrm{SMC}$ fields obtained at the Cerro Tololo Inter-American Observatory 4-m Blanco telescope with the Mosaic II camera attached $\left(36 \times 36 \mathrm{arcmin}^{2}\right.$ field on to a $8 \mathrm{~K} \times 8 \mathrm{~K} \mathrm{CCD}$ detector array). The images are available at the National Optical Astronomy Observatory (NOAO) Science Data Management Archive. ${ }^{1}$ When examining the CMDs of all the catalogued clusters observed in those images, we found 20 intermediate-age or old clusters (Piatti 2011a,b), 69 moderately young or young clusters (in a forthcoming paper), 41 clusters that fall between the image gaps and have not been measured, and 21 candidate star clusters or asterisms. We are here focusing on to the last subgroup of objects. Table 1 lists 11 candidate star clusters along with the main astrometric, photometric and observational information. We recall that the $R$ filter used for imaging has a significantly higher throughput as compared to the standard Washington $T_{1}$ filter, so that $R$ magnitudes can be accurately transformed to yield $T_{1}$ (Geisler 1996). We have also included the log (age) values obtained by C06 and G10 as well as their uncertainty class, following the same notation as used by them: class 1 comprises objects with age errors $\sigma(\log ($ age $))<0.3$; class 2 comprises objects with age errors $0.3 \leq \sigma(\log ($ age $))<0.5$; and class 3 comprises objects with age errors $\sigma(\log ($ age $)) \geq 0.5$. The final information gathered for each candidate star cluster of Table 1 consists of a running number per star, the $x$ and $y$ coordinates, the measured $T_{1}$ magnitudes and $C$ $-T_{1}$ colours, and the observational errors $\sigma\left(T_{1}\right)$ and $\sigma\left(C-T_{1}\right)$. Table 2 gives this information for B119. Only a portion of this table is shown here for guidance regarding its form and content. The full version of Table 2, as well as the final information for the remaining candidate star clusters, is available as Supporting Information with the online version of this paper.

\footnotetext{
${ }^{1}$ http://www.noao.edu/sdm/archives.php.
} 
Table 2. CCD $C T_{1}$ data of stars in the field of B119. This is a sample of the full table, which is available as Supporting Information with the online version of this paper.

\begin{tabular}{lcccccc}
\hline Star & $\begin{array}{c}x \\
\text { (pixels) }\end{array}$ & $\begin{array}{c}y \\
\text { (pixels) }\end{array}$ & $\begin{array}{c}T_{1} \\
(\mathrm{mag})\end{array}$ & $\begin{array}{c}\sigma\left(T_{1}\right) \\
(\mathrm{mag})\end{array}$ & $\begin{array}{c}C-T_{1} \\
(\mathrm{mag})\end{array}$ & $\begin{array}{c}\sigma\left(C-T_{1}\right) \\
(\mathrm{mag})\end{array}$ \\
\hline- & - & - & - & - & - & - \\
10 & 3841.42 & 7168.93 & 20.938 & 0.021 & 0.158 & 0.025 \\
11 & 3841.61 & 7194.83 & 21.242 & 0.043 & 0.565 & 0.046 \\
12 & 3841.88 & 7187.26 & 20.130 & 0.016 & 0.384 & 0.026 \\
- & - & - & - & - & - & - \\
\hline
\end{tabular}

As compared with the data sets used by C06 and G10, the present $C T_{1}$ photometry looks deeper and more accurate. The limiting magnitude of the photometry used by C06 is $V \sim 21.5$ mag. Most and least crowded fields are complete down to $V=20.5 \mathrm{mag}$ at about 80 and 89 per cent levels, respectively. The 50 per cent level is reached at about $V \sim 21.0 \mathrm{mag}$ in the most crowded fields. Inside the radius of the densest clusters, the OGLE data are complete at 80,70 and 50 per cent levels at magnitudes $V=19.0,19.5$ and 20.3, respectively. These $V$ values correspond to MS turn-offs (TOs) of log (age) $\approx 8.3,8.5$ and 8.8, respectively, if the isochrone sets by Girardi et al. $(1995,2002)$, the $E(B-V)$ colour excesses and the $(m-M)_{0}$ distance modulus used by $\mathrm{C} 06$ for the SMC are adopted, independently of the metallicity. On the other hand, the magnitude limit of the photometric survey used by G10 varies as a function of stellar crowding. Zaritsky et al. (2002) found little visible evidence for incompleteness for $V<20 \mathrm{mag}$, corresponding to a MS TO of $\log$ (age) $\approx 8.7$, but the scan edges become visible when plotting the stellar surface density for stars with $20<V<21$ mag. Furthermore, any statistical analysis of this catalogue fainter than $V<20$ mag requires artificial star tests to determine incompleteness, which is becoming significant at these magnitudes. As for the present $C T_{1}$ data set, Piatti (2012) performed different artificial star tests and found that in the most crowded fields the 100 per cent completeness level is reached at $T_{1} \sim 22.0 \mathrm{mag}$, which corresponds to MS TOs of log (age) $\sim 9.6$. MS TOs for stellar populations of 1.4, 2.8 and 8.4 Gyr are placed at $T_{1} \approx 19.9,20.9$ and 21.9 mag, respectively. Thus, we actually reach 1 mag below the MS TO of clusters younger than $\approx 1.5 \mathrm{Gyr}$, which is the age range frequently found for clusters in the SMC inner disc and bar. We conclude that the photometric data sets used by C06 and G10 have been superseded by the present $C T_{1}$ photometric survey.

\section{DATA HANDLING}

The centres and radii of the candidate star clusters are quantities of great importance, since it is possible to confuse a small cluster with a chance grouping of stars in these SMC crowded fields. With this premise in mind, we performed a task as carefully as possible of identification on to the images of the selected objects and then fixed their centres and radii. The whole catalogue by B08 was first mapped on to each image, so that each object in cluster pairs or groups of clusters could be identified without ambiguity. We carried out an interactive routine at the $C$ and $T_{1}$ images for each object separately, making use of the different tools provided by the SAOIMAGE DS $9^{2}$ display program. This display fa-

\footnotetext{
${ }^{2}$ SAOIMAGE DS9 development has been made possible by funding from the Chandra X-ray Science Center (NAS8-03060) and the High Energy Astrophysics Science Archive Center (NCC5-568)
}

cility allowed for multiple frame buffers, region cursor manipulation, many scale algorithms and colour maps, geometric markers, scaling, arbitrary zoom, rotation, pan, a variety of coordinate systems, etc.

Since the appearance of the objects in the images looks like they may contain a handful of comparatively bright stars, or one very bright star surrounded by faint stars, or only relatively faint stars, or a combination of these three possibilities, the stellar density alone could not be a good indicator of the presence of a cluster. For this reason, we think that the above procedure of getting the centres and radii of the studied objects is more meaningful from a physical point of view than performing statistical Gaussian fits on to their star distributions. Figs $1-11$ show $5 \times 5 \operatorname{arcmin}^{2}$ XDSS $B$ images of the selected objects (left-hand panels) and a captured enlargement of the $C$ images centred on the studied candidate star clusters. Only Fig. 1 is shown here as an example; the remaining figures are available as Supporting Information with the online version of this paper. We have drawn black circles with radii as estimated by $\mathrm{B} 08$. The figures present a close-up view of the studied objects. At first glance, some of these small candidate star clusters do not seem to be genuine physical systems. We note that the availability of a less deep photometric data set, like those used by C06 and G10, could possibly mislead the cluster identification. The scale of our images is $0.274 \operatorname{arcsec~pixel}^{-1}$, while those of the OGLE and the Magellanic Clouds Photometric Survey are 0.42 and 0.7 arcsec pixel ${ }^{-1}$, respectively, so that crowding effects at the centre of the objects are more important in their images.

\section{STAR FIELD DECONTAMINATION METHODS}

\subsection{Background}

The issue about the decontamination of cluster fields from field stars has long been treated. Here we only mention some techniques with the aim of summarizing the different approaches considered. Bonatto \& Bica (2007) developed a statistical method that basically involves: (i) dividing the full range of magnitudes and colours of a given CMD into a 3D grid whose cubic cells have axes along the $J$, $J-H$ and $J-K_{\mathrm{s}}$ directions; (ii) computing the expected number density of field stars in each cell based on the number of comparison field stars with magnitudes and colours compatible with those of the cell; and (iii) subtracting randomly the expected number of field stars from each cell. Bonatto \& Bica (2007) and subsequent studies estimate the radius where the subtracted CMD is statistically more significant. With that well-defined CMD, they built a magnitudecolour filter that is applied to the whole photometry, which produces a radial density distribution, if possible, well beyond the cluster limit. Although the method reapplies the cleaning procedure using different cell sizes in the CMDs, they are fixed each time, that is, they do not vary across the CMDs. The latter possibility could be very useful to eliminate field stars with small photometric errors located in CMD regions with a scarce number of stars (e.g. bright red giants, etc.).

Pavani \& Bica (2007) and Pavani et al. (2011), based on the Bonatto \& Bica (2007) method, further adapted the $R^{2}$ statistical test to uncover possible open cluster remnants, for which CMD field decontamination is priority. They tested whether the remnant candidate can be reproduced by equal area field fluctuations sorted out in the background area. This method is very suitable 


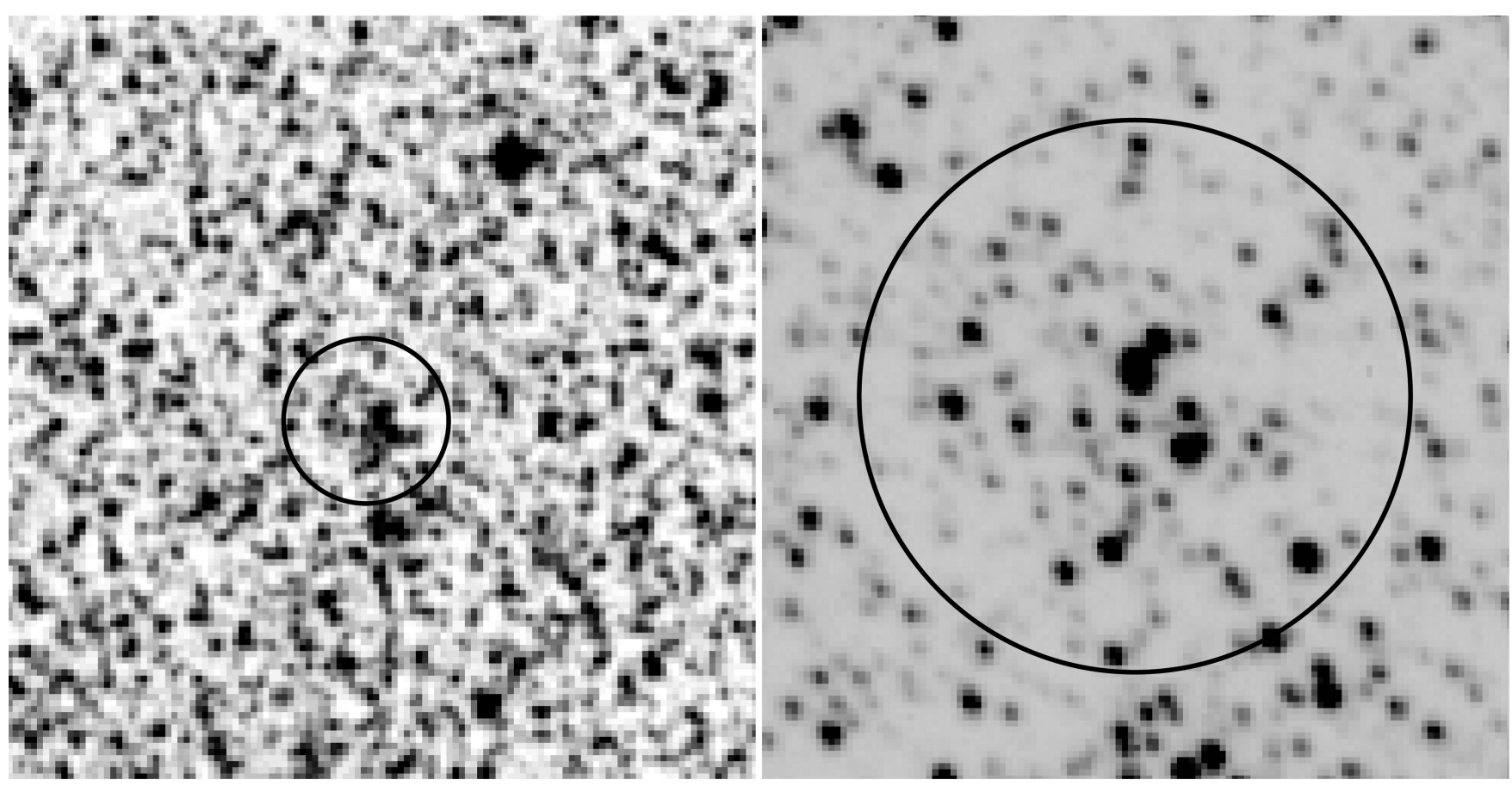

Figure 1. $5 \times 5 \operatorname{arcmin}^{2}$ XDSS $B$ image centred on B119 (left-hand panel). North is up and east is to the left. The superimposed circle corresponds to the radius adopted by Bica et al. (2008). An enlargement of our $C$ image centred on the object is shown in the right-hand panel.

for sparsely populated objects. Maia et al. (2010) also followed the precepts by Bonatto \& Bica (2007) to develop their own decontamination procedure. As above, they used cells in the CMDs with constant colour and magnitude bins for each size and positional configuration. On the one hand, they created an exclusion index by noting how many times each star was removed from the sample, and then normalizing by the number of grid configurations. On the other hand, they computed an average membership for each star. The decontamination procedure consists of applying complementarily both indicators. They imposed the restriction that the field CMD should have the same reddening as the cluster CMD.

With the aim of estimating a magnitude from which the characteristics of the observed field stars are indistinguishable from those of the clusters in terms of spatial density, magnitude and colour distributions, Piatti, Clariá \& Ahumada (2010) applied a statistical method to filter the field stars from the CMDs and from the colour-colour diagrams. They divided the observed region into boxes with the same number of pixels per side, and built for each of them the corresponding CMDs. At first glance, the method appears profitable when some differences can be seen between the various box-extracted CMDs, a fact which reveals a lack of homogeneity in the spatial distribution of the brightness and colours of the stars. The method used consists of alternatively adopting any of the box-extracted 'reference' CMDs to statistically filter the remaining ones. This filtering task was repeated using each of the box-extracted CMDs as a reference CMD. At the end of this process, each box-extracted CMD was individually filtered, using each time a different reference CMD. The filtering was performed in such a way that the stars in different magnitude-colour bins for each reference CMD are subtracted from the remaining CMDs by removing those stars closer in magnitude and colour to the ones of the reference CMD. When comparing the various filtered CMDs corresponding to a given box with the observed one, the residuals from box-to-box variations and the fiducial CMD features of that box are found. This is due to the fact that a star that has magnitude and colours within the typical values found in the reference field CMDs is in most cases eliminated. Thus, the fewer number of times a star is removed in a given box, the larger its probability of representing a fiducial feature in that box. Piatti et al. (2010) adopted any star that was removed fewer than 20 per cent of the times as a probable fiducial feature star. They found in this way a magnitude from which it is hardly possible to distinguish cluster stars from field stars.

\subsection{An alternative cleaning procedure}

From our experience in cleaning the field star contamination in the cluster CMDs, we have identified some situations which still need our attention. It frequently happens that some parts of the CMDs are more populated than others, so that fixing the size of the cells in the CMDs becomes a difficult task. Small cells do not usually carry out a satisfactory job in CMD regions with a scarce number of fields stars, while big cells fail in populous CMD regions. Thus, relatively bright field red giants with small photometric errors could not be subtracted and, consequently, the cluster CMD could show spurious red giant features. A compromise between minimizing the residuals left after the subtraction of field stars from the cluster CMDs and maximizing the cleaning of field stars is always desirable. This fact has led previous procedures to try with different cell sizes as a general budget which, in turn, depends on the sizes of the spatial regions used to build the extracted CMDs. Sometimes the radial density profile of a star cluster is taken into account to assign statistical membership probabilities. This constrains the star field cleaning procedure to those clusters for which it is possible to satisfactorily trace their stellar radial distributions. Unfortunately, this is not the case for objects with a handful of stars or of small angular dimensions.

We have designed an alternative procedure which makes use of variable cells in the CMDs. Magnitude and colour cell sizes are 
varied separately. The cells are adjusted in such a way that they result bigger in CMD regions with a scarce number of stars, and vice versa. This way we pursue to map the field CMD as closely as possible on to the cluster CMD. The method does not need to know whether a star is placed close to the cluster centre, nor the cluster radial density profile to infer a membership probability. However, it takes into account the star field density, since the more populous a star field, the larger the number of stars subtracted from the cluster CMD. This is accomplished by eliminating one star - the closest one in terms of magnitude and colour - in the cluster CMD for each star identified in the field CMD. As a result, the intrinsic spatial star distribution is uncovered within the object region.

Once the field CMD is adopted, the method defines a free path for each star as the distance to the closest star in the field CMD. Magnitude and colour directions are separately considered, so that $(\Delta(\text { colour }))^{2}+(\Delta$ (magnitude $\left.)\right)^{2}=(\text { free path })^{2}$, where $\Delta$ (colour) and $\Delta$ (magnitude) are the distances from the considered star to the closest one in abscissa and ordinate in the field CMD. The method has shown to be able to eliminate stochastic effects in the cluster CMDs caused by the presence of isolated bright stars, as well as to make a finer cleaning in the most populous CMD regions. In order to prevent from large non-meaningful free paths, the method imposes a reasonably large free path limit. The free path of a field star accounts for a zone of influence (rectangle) of that star in the $\mathrm{CMD}$, in the sense that only the closest star inside that area in the cluster CMD is eliminated.

For our list of candidate star clusters we cleaned circular regions centred on the objects with radii twice as large as those estimated by B08 for the objects, that is, we cleaned areas four times bigger than those of the circles of Figs 1-12. As for the reference star fields, we used four different regions per object with the same area as for the candidate clusters. These circular field areas were placed towards the north, east, west and south of the candidate cluster at a distance of four times the radii of the circles of Figs 1-12. This was done in order to take into account variations in the spatial density, magnitude and colours of field stars. Note that the candidate star clusters are of small angular size, typically $\sim 0.1-0.3$ arcmin. We previously performed a large number of tests by using as object and field CMDs a unique selected field, or selecting two different field CMDs, one of them acting as a cluster CMD. The results of our various experiments showed that residuals are not left or they are minimal. Anyway, we do not rule out more noticeable residuals due to the non-uniformity of the field in terms of spatial density, colour distribution and luminosity function. However, whenever an excess of stars remains in the cleaned CMDs, we assume that we are dealing with an enhancement of stars caused by the presence of a possible star cluster or by a stellar fluctuation in the SMC field or by a chance grouping of stars.

The method was run four times for each studied object, each time using a different reference field area. Thus, we obtained four different cleaned CMDs per object. When comparing those CMDs, one may find stars that were not subtracted for most of the times, while other stars were subtracted in most of the program executions. The different numbers of times that a star keeps unsubtracted can then be converted in a measure of the probability of being a fiducial feature of the candidate cluster field. Thus, we are able to distinguish stellar populations projected on to cluster fields that have a probability $P$ $<25$ per cent of being genuine candidate cluster populations, that is, a typical foreground population of stars that could indistinguishably belong to the star field or to the studied object $(P=50$ per cent) and of stars that are predominantly found towards the can- didate cluster field $(P>75$ per cent) rather than in the star field population.

\subsection{Results}

Figs 12-22 show in the top right-hand panels the extracted field CMDs. Only Fig. 12 is shown here as an example; the remaining figures are available as Supporting Information with the online version of this paper. Instead of plotting the position of each field star, the graphics present rectangles - which come from the definition of a free path for each star - centred on those positions, with the aim of illustrating how the technique works. As can be seen, this technique allows using smaller and larger rectangles according to the colour and magnitude frequencies in the CMDs. We think that the flexibility provided by variable cells can reproduce more tightly the field CMD features on to the candidate cluster CMDs than those of fixed size, thus allowing improvements in the field star cleaning process. The top left-hand panels depict schematic finding charts including all the measured stars drawn using the open circles, whose sizes are proportional to the $T_{1}$ brightness of the stars. The stars with the filled black, dark-grey and clear-grey circles have probabilities of being an intrinsic candidate cluster feature higher than 75 per cent, equal to 50 per cent and lower than 25 per cent, respectively. The circle represents the adopted radius which, in some cases, is slightly shifted or resized as compared to that given in B08. This was done in order to encompass stars that better define the field CMD features. The bottom panels of the figures depict stars located within the overplotted circles for three different probability regimes. The composite CMD from these three CMDs results in the observed candidate cluster CMD.

By comparing the three bottom CMDs we can find out whether a sequence or group of stars come from the superposition of different field populations or is part of the candidate cluster population. Some hints at this stage are easy to recognize. We have overplotted on to the bottom panels of Figs 12-22 the isochrones adopted by C06 and G10, drawn with the solid and dashed lines, respectively. We have used their metallicities, $E(B-V)$ colour excesses and SMC distance moduli. For comparison purposes, we have also included the zero-age main-sequences (ZAMSs, $Z=0.008$, Piatti 2011b) with the solid lines in all the CMDs, using a SMC distance modulus of $m-M=18.9 \pm 0.1$ and the $E(B-V)$ colour excesses taken from the Burstein \& Heiles (1982, hereafter BH82) extinction map. Whenever possible, we also estimate the cluster ages by fitting the isochrones of Girardi et al. (2002), which are shown with the dotted lines. We provide below with details about the studied candidate star clusters:

(i) B119. The $P(<25$ per cent $)$ CMD shows the oldest field population that we find along the line of sight towards the object. According to the $\delta\left(T_{1}\right)$ index - which measures the difference in $T_{1}$ magnitudes between the red clump (RC) and the MS TO - and equation (4) from Geisler et al. (1997), its age is $\sim 2.5-3.0$ Gyr. Note that some hints of such an evolved population are also seen in the $P(=50$ per cent $)$ and $P(>75$ per cent $)$ CMDs. These CMDs also show a relatively straight MS which does not resemble that of a moderately young cluster. Since multiple populations appear in the three CMDs, we do not classify this object as a possible cluster.

(ii) $B S 20 . \mathrm{RC}$ stars seem to appear in the three bottom CMDs. These stars are placed at $T_{1} \approx 18.0 \pm 0.5 \mathrm{mag}$. We discard the possibility of an intermediate-age cluster $(t>1 \mathrm{Gyr})$, since we had expected to see its RC at a lower $T_{1}$ magnitude. For instance, 

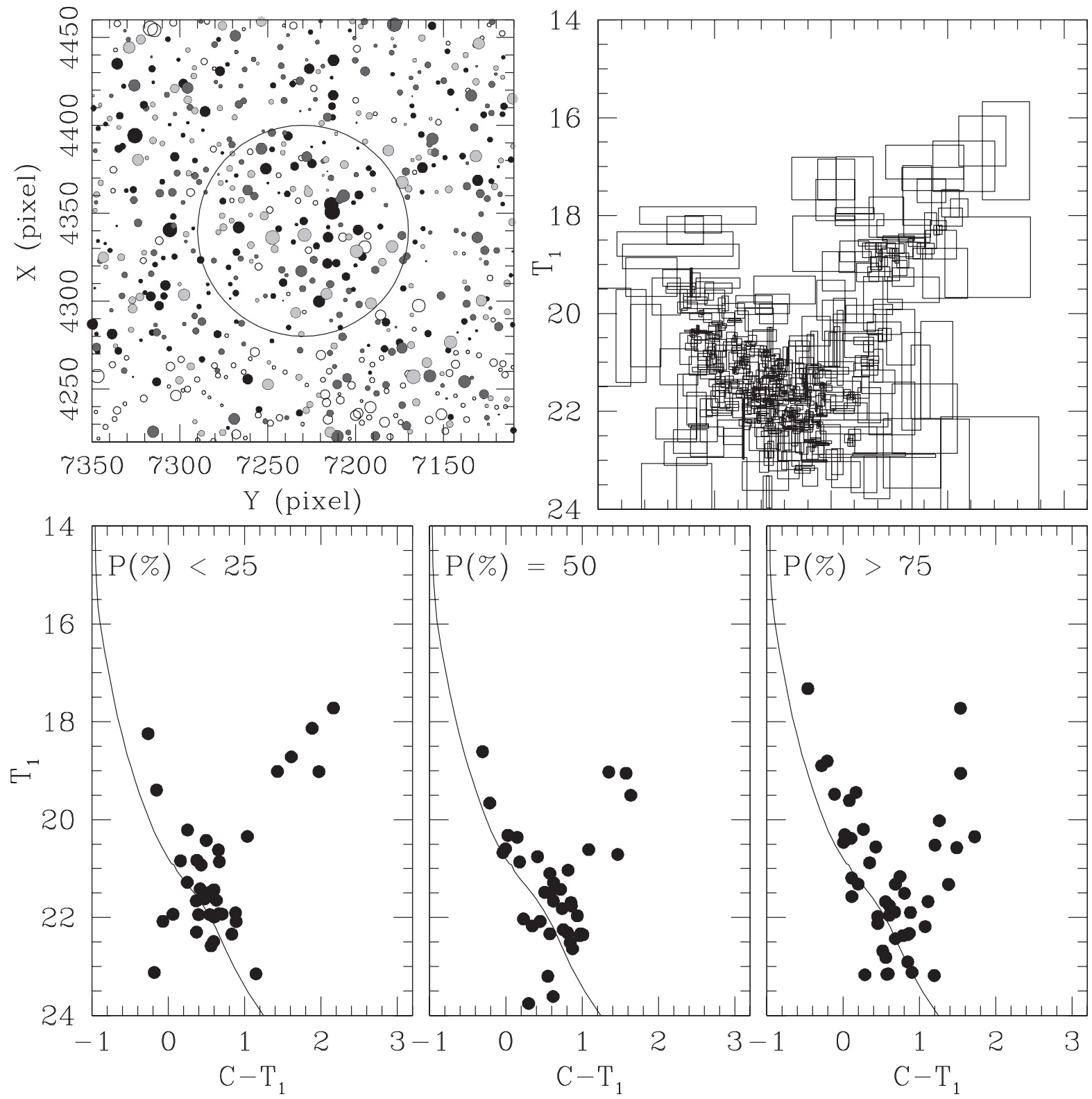

Figure 12. Schematic finding chart of the stars measured in the field of B119 (upper left-hand panel), with a circle corresponding to the adopted radius. North is up and east is to the left. The size of the symbols is proportional to the $T_{1}$ magnitude. The filled circles correspond to stars with a probability of being a feature of the cluster field higher than 75 per cent. The equal cluster area field CMD is shown at the top right-hand panel, wherein the stars are placed at the centres of their respective free path rectangles. For stars inside the candidate cluster radius, three different CMDs are shown, distinguishing those stars that have chances of being a candidate cluster field feature $<25$ per cent, equal to 50 per cent and $>75$ per cent, respectively. The ZAMS (solid line) and the isochrones from Girardi et al. (2002) for the ages derived by C06 (solid line) and G10 (dashed line) are superimposed (see details in Section 4).

RC stars older than 1.0 Gyr are placed at $T_{1} \sim 18.8-19.1 \mathrm{mag}$, depending on the stars' positions due to the galaxy depth (Crowl et al. 2001). In this case, the cluster would be far away from the SMC limits, between the SMC and our Galaxy. In addition, if we adopt $T_{1} \sim 18.2 \mathrm{mag}$ for the cluster MS TO, then the $\delta\left(T_{1}\right)$ index turns out to be $\gtrsim 2.3$, which implies an age older than $3.5 \mathrm{Gyr}$. It is hardly possible that BS20 is an old SMC cluster (Piatti 2011b). Another possibility is that the object is a moderately young cluster, if we assume that the RC stars belong to the object. In such a case, an isochrone of $\log (t)=8.55[E(B-V)=0.035, Z=0.008]$ would fit the RC stars better than C06 and G10. We might be dealing with a chance grouping of stars composed by a handful of faint MS stars and a few RC field stars. (iii) $B S 25$. The $P(>75$ per cent $)$ and $P(<25$ per cent $)$ CMDs are almost identical, with the only difference being the few stars brighter than $T_{1}<20$ mag and bluer than $C-T_{1}<0.2$ mag. Old field MS stars $\left(T_{1}>21 \mathrm{mag}\right)$ contaminate the entire candidate cluster field, as judged by the presence of them in the three bottom CMDs. Note also that a possible subgiant branch at $T_{1}=20$ is also visible. On the other hand, the $P(=50$ per cent) CMD shows a straight MS which does not match the lower envelope of any young cluster MS. Based on the present observational limitations, we are inclined to conclude that we are dealing with a possible overdensity of faint stars, with superimposed few brighter sources.

(iv) $B S 35$. As far as the stellar populations in the three bottom CMDs are compared, we note that the star fields far outside from 
and inside the object circle are distinguishable. The cluster appears to be slightly younger than what C06 and G10 estimated. Indeed, the isochrone which best reproduces the $P(>75$ per cent $)$ CMD's features is that of $\log (t)=8.70 \pm 0.05[E(B-V)=0.014, Z=$ 0.008]. Note that the MS TOs of the isochrones adopted by $\mathrm{C} 06$ and G10 are redder than that for the object.

(v) BS251. At first glance, it would appear that the small angular size, low-density candidate cluster is projected on to a much older and denser star field, in very good agreement with the age obtained by C06. However, the space distribution of stars with $P(>75$ per cent $)$ and $P(=50$ per cent $)$ is not as concentrated as that for the $P(<25$ per cent $)$ stars. Based on this feature, we conclude that they are rather a star fluctuation in the field.

(vi) BS265. The cluster arises clearer in the $P(>75$ per cent $)$ CMD where we fitted an isochrone for $\log (t)=8.8 \pm 0.1$, as $\mathrm{G} 10 \mathrm{did}$, but using a different metallicity value $(Z=0.008)$. The $E(B-V)$ obtained from $\mathrm{BH} 82$ is $0.068 \mathrm{mag}$.

(vii) H86-70. This is one of the cases where the cluster appears projected on to a star field blurring its CMD's fiducial features. However, the cleaning procedure allows us to disentangle them. The bottom left-hand panel shows a mixture of younger and older field MS stars and RC field stars, while the bottom right-hand panel depicts a clear relatively tight cluster MS and, presumably, some cluster RC stars. Since field and cluster stars appear to have similar ages, C06 and G10 estimated a cluster age in excellent agreement with our present estimate $[\log (t)=8.8 \pm 0.1, E(B-V)=0.035$, $Z=0.008]$.

(viii) H86-78s. This is a small angular size candidate cluster composed of a few bright stars, since they only appear in the $P(>75$ per cent) CMD; the star field is seen in the three bottom CMDs and is clearly older. Since the two apparent lower cluster MS stars in the $P(>75$ per cent $)$ CMD are likely field stars, we conclude that this object is a chance grouping of stars.

(ix) H86-86. Despite the visible excess of subgiant and red giant stars in the three bottom panels - which resembles older field SMC populations - the positions of the MS stars are reproduced by an isochrone of $\log ($ age $)=8.1$ (G10) satisfactorily. Note, however, that such MS stars are not mainly seen in the $P(>75$ per cent $)$ CMD. We are possibly dealing with a chance grouping of stars. Therefore, the isochrone fitting performed by G10 would represent the age of the younger stellar population seen towards this line of sight.

(x) H86-88. The catalogued object does not result to be a clear star cluster but rather a chance grouping of a few bright and a handful of low MS field stars. The composite field CMD features led C06 and G10 to fit very different isochrones to the observed CMD.

(xi) H86-197. We confirm the physical reality of this intermediate-age cluster located in the outer SMC disc. This is for the first time that an age estimate in provided for the object. We find that the isochrones that best resemble the cluster CMD feature are that for $\log (t)=9.1 \pm 0.1[E(B-V)=0.024, Z=0.008]$.

Our results suggest that nearly one-third of the studied candidate star clusters would appear to be genuine physical systems. In addition, we find in most of the studied objects that there are more stars with $P(>75$ per cent $)$ outside the cluster circle than inside. The photometric errors of stars with probabilities of being a fiducial feature of the candidate cluster field higher than 75 per cent are at most twice as big as the plotted filled circles in $C-T_{1}$ colour and smaller than the filled circles in $T_{1}$ magnitude. This means that the colour dispersion observed in some cleaned CMDs comes from an intrinsic dispersion in the colours of the involved stars. On the other hand, our study shows that the ages derived by $\mathrm{C} 06$ and G10 can reflect those of the composite stellar populations of the SMC field. As far as we are aware of, this is for the first time that evidence is presented showing that some SMC candidate star clusters are not possibly genuine physical systems. Additionally, we have studied other 10 candidate star clusters (B110, B112, BS38, H86-78n, H86-91, H86-95, H86-96, H86-98, H86-103, H86-121), following the same precepts as described for objects of Table 1, and also found that they are possible non-clusters. However, we cannot draw final conclusions on their status since the images from which the photometry was obtained contain some saturated stars.

The resulting possible non-cluster sample represents nearly 10 per cent of the 152 objects placed throughout the 11 observed SMC fields (see Section 2). This does not seem to be a significant percentage of the catalogued clusters. If we assume a similar percentage for the whole catalogued SMC clusters (Piatti 2011b), we would expect that some $\sim 60$ objects were not real stellar aggregates. However, since these objects have their own spatial distribution, it would be interesting to recover the expected spatial distribution of genuine star clusters as a perspective exercise. Thus, the spatial distribution of possible non-clusters is used to correct that of the clusters in the observed 11 SMC fields in order to obtain an intrinsic spatial distribution. Then, by assuming that the area covered by these 11 Mosaic II fields represents an unbiased subsample of the SMC as a whole, the expected spatial distribution of the SMC cluster system is obtained, once it is statistically decontaminated by the spatial distribution of the possible non-clusters.

Viewing the SMC as a triaxial galaxy with the declination (Dec.), right ascension (RA) and line of sight as the three axes, Crowl et al. (2001) found axial ratios of approximately 1:2:4. Based on this result, and with the purpose of describing the spatial distribution of the clusters, we decided to use an elliptical framework instead of a spherical one in order to reflect more meaningfully the flattening of the galaxy (Piatti 2011b) where $a$ is the semimajor axis - parallel to the SMC main body - of an ellipse centred at RA = $00^{\mathrm{h}} 52^{\mathrm{m}} 45^{\mathrm{s}}$, Dec. $=72^{\circ} 49^{\prime} 43^{\prime \prime}(\mathrm{J} 2000)($ Crowl et al. 2001) and with a b/a ratio of $1 / 2$. Thus, we assume that the cluster spatial distribution correlates much better with a pseudo-elliptical (projected) distance measured from the galaxy centre than with the radial distance, or distances defined along the RA or Dec. axis. For the subsequent analysis, we adopt the semimajor axis $a$ as the representative spatial variable to trace the behaviour of the cluster spatial distribution throughout the galaxy.

We computed for each cluster in B08 the value of $a$ that an ellipse would have if it were centred on the SMC centre, had a $b / a$ ratio of $1 / 2$ and one point of its trajectory coincided with the cluster position. We assume for the SMC the limits quoted by B08. We counted the number of clusters in elliptical rings from $a=0^{\circ}$ up to $8^{\circ}$. The size of the rings was varied from 0.05 up to 0.5 , so that we built a total of 10 cluster spatial distributions. Binning data where the distribution changes steeply frequently have biases in the measured distribution. In this case, it tends to pull the central spatial distribution remarkably high, since more clusters migrate into a given bin from an adjacent bin where there are more clusters than from the other adjacent bins where there are fewer clusters. The errors increase as one goes outside-in towards the SMC centre. We averaged the 10 resulting spatial distributions in order to mitigate the influence of any chosen bin size. We repeated the same procedure to build the spatial distribution of non-possible clusters as well as that for the observed ones in the 11 studied SMC fields. Then, we calculated the expected number of clusters in the whole galaxy, 


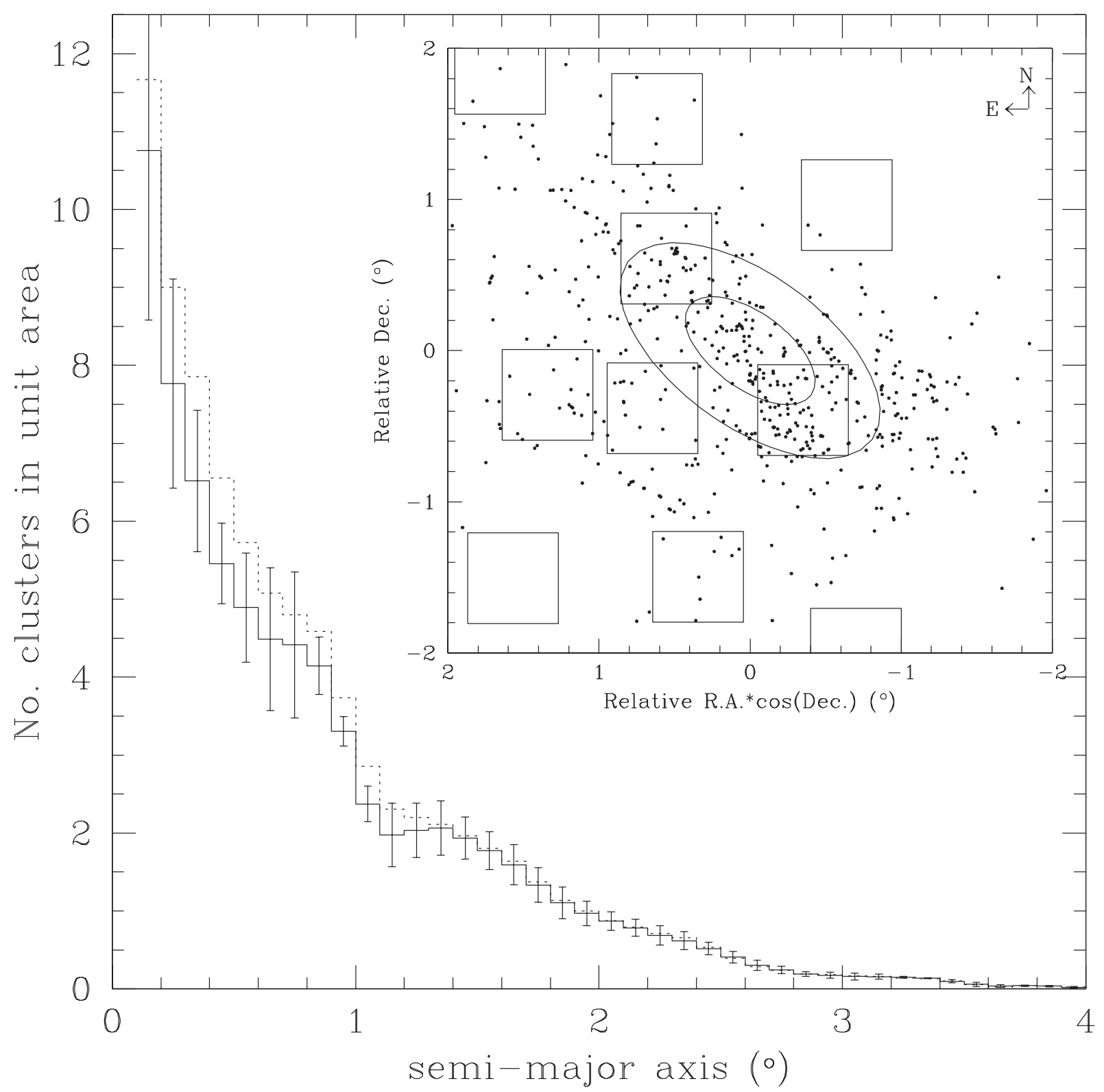

Figure 23. Spatial distribution of SMC clusters.

corrected for possible asterisms in B08's catalogue according to the expression

$N_{\text {total }}=\left(N_{\text {obs }}-N_{\text {nocls }}\right) N_{\text {cat }} / N_{\text {obs }}$,

where $N_{\text {nocls }}, N_{\text {obs }}$ and $N_{\text {cat }}$ represent the spatial distributions of possible non-clusters, of observed clusters in the $11 \mathrm{SMC}$ fields and of catalogued clusters in B08, respectively.

The inner panel of Fig. 23 depicts the B08 cluster distribution (dots) and two ellipses of $a=0.5$ and 1.0. The large boxes represent the studied Mosaic II fields. One of them, centred on the cluster $\mathrm{AM} 3$, falls beyond the figure limits at (relative RA $\times \cos (\mathrm{Dec}$.), relative Dec.) $\approx\left(-4.7,0.0^{\circ}\right)$. The larger panel shows both cluster spatial distributions, the one directly obtained from B08 (dotted histogram) and that decontaminated from possible non-clusters (solid histogram). The error bars represent a measure of the effect caused by choosing different bin sizes when building the cluster spatial distribution. As can be seen, there is no clear difference between expected and observed cluster spatial distributions within $1 \sigma$, although it would become significant (a histogram difference larger than $2 \sigma$ ) between $a \approx 0.3$ and 1.2 , if the asterisms were increased up to 20 per cent.

\section{SUMMARY}

To date, the catalogue by B08 has been the most complete compilation of star clusters in the SMC. Most of these objects have not been studied yet. Here, we present for the first time Washington $C T_{1}$ photometry for 11 unstudied or poorly studied candidate star clusters. As compared with the data sets from previous photometric surveys, the present $C T_{1}$ photometry turns out to be deeper and more accurate. In general, the selected objects appear to be of small angular size and contain a handful of stars. They are projected towards the most crowded star field regions in the SMC, at distances shorter than $\sim 1^{\circ}$ from its centre. 
We have designed a procedure for cleaning the cluster CMDs from the unavoidable star field contamination which makes use of variable cells in the CMDs. The cells are adjusted in such a way that they result bigger in CMD regions with a scarce number of field stars, and vice versa. This way, we reproduce the field CMD as closely as possible on to the cluster CMD. The method does not need to know whether a star is placed close to the cluster centre, nor the cluster radial density profile to infer a membership probability. However, it takes into account the star field density, since the more populous a star field, the larger the number of stars subtracted from the cluster CMD. As a result, the intrinsic spatial star distribution is uncovered within the object region. Once the field CMD is adopted, the method defines a free path for each field star as the distance to the closest star in the field CMD. The method has shown to be able to eliminate stochastic effects in the cluster CMDs caused by the presence of isolated bright stars, as well as to make a finer cleaning in the most populous CMD regions.

When applying the cleaning procedure to the CMDs of the 11 selected candidate star clusters, we found that nearly one-third of them would appear to be genuine physical systems. We estimated their ages from the matching of the isochrone which best reproduces the CMD cluster features. In this sense, the ages previously derived for some of them mostly reflect those of the composite stellar populations of the SMC field. The present analysis tools applied to faint poorly populated clusters or candidates in the Magellanic Clouds point to the need of better scale deep observations with, for example, 8-m class telescopes.

Finally, we used the spatial distribution in the SMC of possible non-clusters to statistically decontaminate that of the SMC cluster system. By assuming that the area covered by 11 studied fields $\left(36 \times 36 \operatorname{arcmin}^{2}\right.$ each $)$ represents an unbiased subsample of the SMC as a whole and by using an elliptical framework centred on the SMC centre $(b / a=1 / 2)$, we found that there is no significant difference between the expected and observed cluster spatial distributions. However, a difference at a $2 \sigma$ level would become visible between $a \approx 0.3$ and 1.2 , if we doubled the amount of possible non-clusters.

\section{ACKNOWLEDGMENTS}

We greatly appreciate the comments and suggestions raised by the reviewer which helped us to improve the manuscript. This research draws upon data distributed by the NOAO Science Archive, and has made use of SAOIMAGE DS9, developed by the Smithsonian Astrophysical Observatory. NOAO is operated by the Association of Universities for Research in Astronomy (AURA), Inc., under a cooperative agreement with the National Science Foundation. This work was partially supported by the Argentinian institutions
CONICET and Agencia Nacional de Promoción Científica y Tecnológica (ANPCyT), and the Brazilian Agency CNPq.

\section{REFERENCES}

Bica E., Schmitt H. R., 1995, ApJS, 101, 41

Bica E., Bonatto C., Dutra C. M., Santos J. F. C., Jr, 2008, MNRAS, 389, 678 (B08)

Bonatto C., Bica E., 2007, MNRAS, 377, 1301

Bruck M. T., 1975, MNRAS, 173, 327

Burstein D., Heiles C., 1982, AJ, 87, 1165 (BH82)

Chiosi E., Vallenari A., Held E. V., Rizzi L., Moretti A., 2006, A\&A, 452, 179 (C06)

Crowl H. H., Sarajedini A., Piatti A. E., Geisler D., Bica E., Clariá J. J., Santos J. F. C., Jr, 2001, AJ, 122, 220

Geisler D., 1996, AJ, 111, 480

Geisler D., Bica E., Dottori H., Clariá J. J., Piatti A. E., Santos J. F. C., Jr, 1997, AJ, 114, 1920

Girardi L., Chiosi C., Bertelli G., Bressan A., 1995, A\&A, 298, 87

Girardi L., Bertelli G., Bressan A., Chiosi C., Groenewegen M. A. T., Marigo P., Salasnich B., Weiss A., 2002, A\&A, 391, 195

Glatt K., Grebel E. K., Koch A., 2010, A\&A, 517, 50 (G10)

Hodge P. W., 1986, PASP, 98, 1113

Maia F. F. S., Corradi W. J. B., Santos J. F. C., Jr, 2010, MNRAS, 407, 1875

Pavani D. B., Bica E., 2007, A\&A, 468, 139

Pavani D. B., Kerber L. O., Bica E., Maciel W. J., 2011, MNRAS, 412, 1611

Piatti A. E., 2011a, MNRAS, 416, L89

Piatti A. E., 2011b, MNRAS, 418, L69

Piatti A. E., 2012, MNRAS, 422, 1109

Piatti A. E., Clariá J. J., Ahumada A. V., 2010, PASP, 122, 516

Pietrzynski G., Udalski A., Kubiat M., Szymanski M., Wozniak P., Zebrun K., 1999, Acta Astron., 49, 521

Udalski A., Szymanski M., Kubiak M., Pietrzynski G., Wozniak P., Zebrun K., 1998, Acta Astron., 48, 147

Zaritsky D., Harris J., Thompson I. B., Grebel E. K., Massey P., 2002, AJ, 123,855

\section{SUPPORTING INFORMATION}

Additional Supporting Information may be found in the online version of this article:

Table 2. CCD $C T_{1}$ data of stars in the field of B119.

Figs 2-11. Images of the selected objects.

Figs 13-22. Finding charts and CMDs for candidate clusters.

Please note: Wiley-Blackwell are not responsible for the content or functionality of any supporting materials supplied by the authors. Any queries (other than missing material) should be directed to the corresponding author for the article.

This paper has been typeset from a $\mathrm{T}_{\mathrm{E}} \mathrm{X} / \mathrm{L} \mathrm{T} \mathrm{E} \mathrm{X}$ file prepared by the author. 